\title{
Natural orifice transluminal endoscopic surgery and transrectal abscess drainage: Is this what we are getting into?
}

\author{
Lawrence C Hookey BSc MD
}

$\mathrm{N}$ atural orifice transluminal endoscopic surgery (NOTES) has advanced dramatically over the past year. Research teams are starting to publish results more frequently (90 articles to date in 2008, 73 in 2007, and 15 in 2006). The scientific method is now being applied to ideas in an effort to refine any technique in animal models before embarking on its use in humans. That being said, there are several ongoing trials of hybrid NOTES/laparoscopic surgeries in human subjects. There continues to be major interest from members of the medical device industry with further research funding available through NOTES-specific associations and prototype development well underway by these key players in endotherapy. However, there are some key issues yet to be addressed with satisfaction, including:

- the development of NOTES-specific endoscopes, capable of deflection in several directions, also capable of applying traction in one direction while cutting in another direction;

- quantification of the infection risk associated with crossing natural orifices and barriers and the necessary steps to prevent infection risks; and

- orientation of the scope tip in the peritoneum as well as a method of knowing where the scope body is, thereby reducing the risk of inadvertent trauma to 'bystander organs' $(1,2)$.

While it may seem that NOTES has advanced by leaps and bounds, it has actually been in development for many years and continues to steadily progress with each study addressing these key questions and others. A core philosophy of the North American NOTES association - the Natural Orifice Surgery Consortium for Assessment and Research (NOSCAR) - is that all human NOTES procedures be performed under the supervision of a research ethics board and preferably within a research protocol (1). This is an effort to prevent, or at least discourage, 'one-off' procedures which, while interesting, are difficult to translate to the greater body of literature or to general NOTES practice.

This brings us to the case report in this month's Journal by Alis et al (pages 983-986). They report an endoscopic transrectal drainage of an appendiceal abscess using a needle knife and sphincterotome without the aid of catheters or stents. While interesting in its own right, the authors tie this intervention in with NOTES, stating that it is another application within this evolving arena. Although I personally do not believe the case would qualify as a NOTES procedure (the lumen was incised but the scope never passed through the colonic wall, nor was there an effort to close the hole or perform any further intervention in the peritoneum), let us consider it for a moment as one. As stated above, human NOTES interventions should ideally be performed under the approval of a research ethics board and within a broader protocol. If one is to report such cases as NOTES-related, one should be held to the same standards.

As stated above, the difficulty with the report by Alis et al is relating it to the care of other patients. An important detail about this particular case is the patient's age -16 years. He tolerated the procedure well and recovered quickly, both of which were at least partially attributable to his age and health status. Whether an older patient with significant comorbidities would have fared as well is questionable. Furthermore, the authors did not provide information on any follow-up endoscopy and whether the incision had left any visible scarring or opening. It certainly would not fall within the usual standard of care for a pelvic abscess (hence its publication) and it is easily conceivable that the procedure could have gone much more poorly - for instance, there could have been intra-abdominal dissemination of the abscess contents with worsening sepsis. So, while the paper by Alis et al is interesting, it is difficult to suggest any change in current practice based on their report given the questions that surround its potential use in other patients.

The field of NOTES is advancing rapidly and gaining traction as a feasible, desirable method of minimally invasive surgery $(3,4)$. However, to advance our knowledge and ensure our patients' safety, we need to strive to maintain the highest standards of research methodology and ethics in our research in this field, where one avoidable adverse event could have repercussions far beyond those for the individual patient.

\section{REFERENCES}

1. ASGE/SAGES Working Group on Natural Orifice Translumenal Endoscopic Surgery White Paper October 2005. Gastrointest Endosc 2006;63:199-203.

2. McGee MF, Rosen MJ, Marks J, et al. A primer on natural orifice transluminal endoscopic surgery: Building a new paradigm. Surg Innov 2006;13:86-93.

3. Swain P. A justification for NOTES - natural orifice translumenal endosurgery. Gastrointest Endosc 2007;65:514-6.

4. Varadarajulu S, Tamhane A, Drelichman ER. Patient perception of natural orifice transluminal endoscopic surgery as a technique for cholecystectomy. Gastrointest Endosc 2008;67:854-60.

\footnotetext{
Hotel Dieu Hospital, Division of Gastroenterology, Department of Medicine, Queen's University, Kingston, Ontario

Correspondence: Dr Lawrence C Hookey, 166 Brock Street, Hotel Dieu Hospital, Kingston, Ontario K7L 5G2.

Telephone 613-544-3310, fax 613-544-3114, e-mail hookey@hdh.kari.net

Received and accepted for publication July 10, 2008
} 


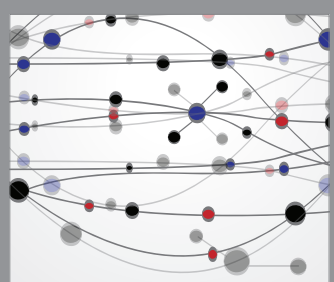

The Scientific World Journal
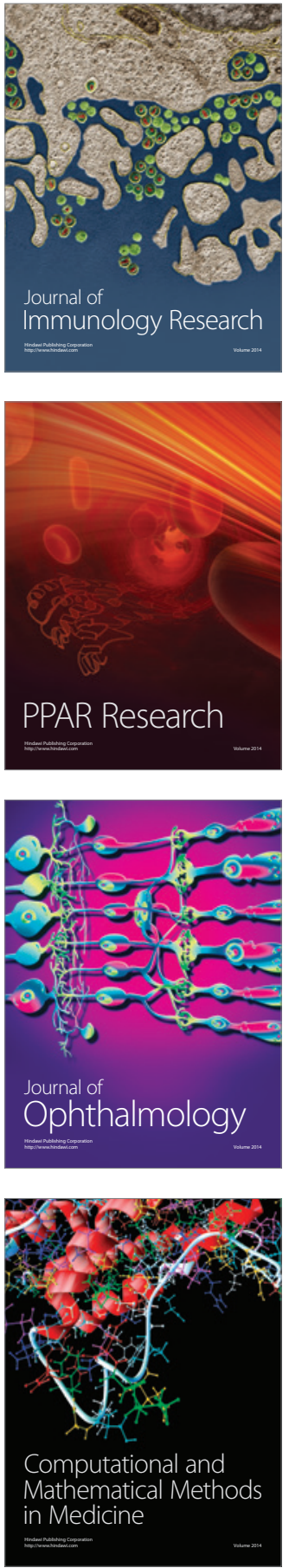

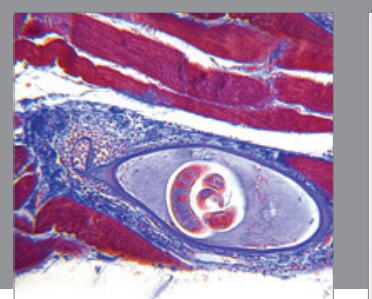

Gastroenterology Research and Practice

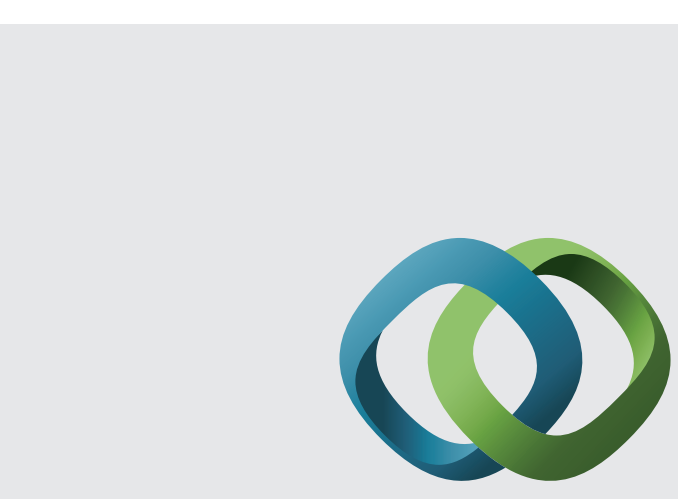

\section{Hindawi}

Submit your manuscripts at

http://www.hindawi.com
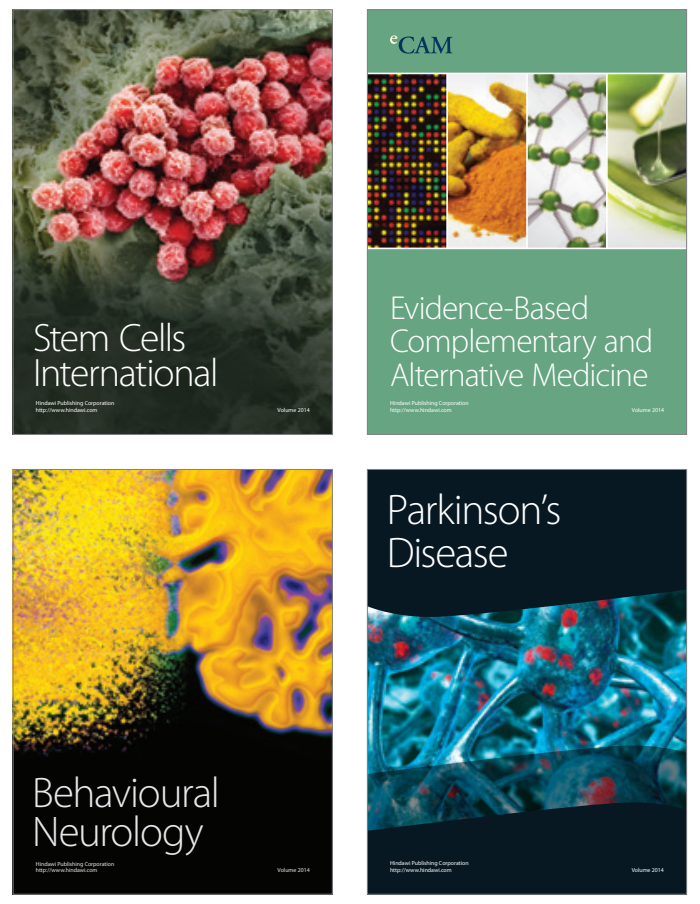
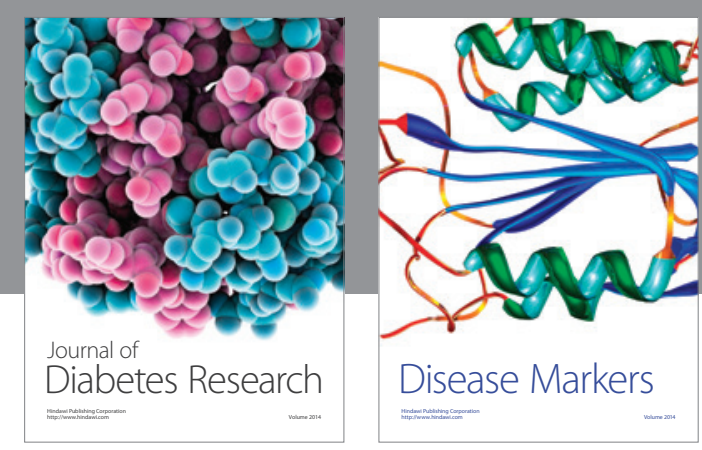

Disease Markers
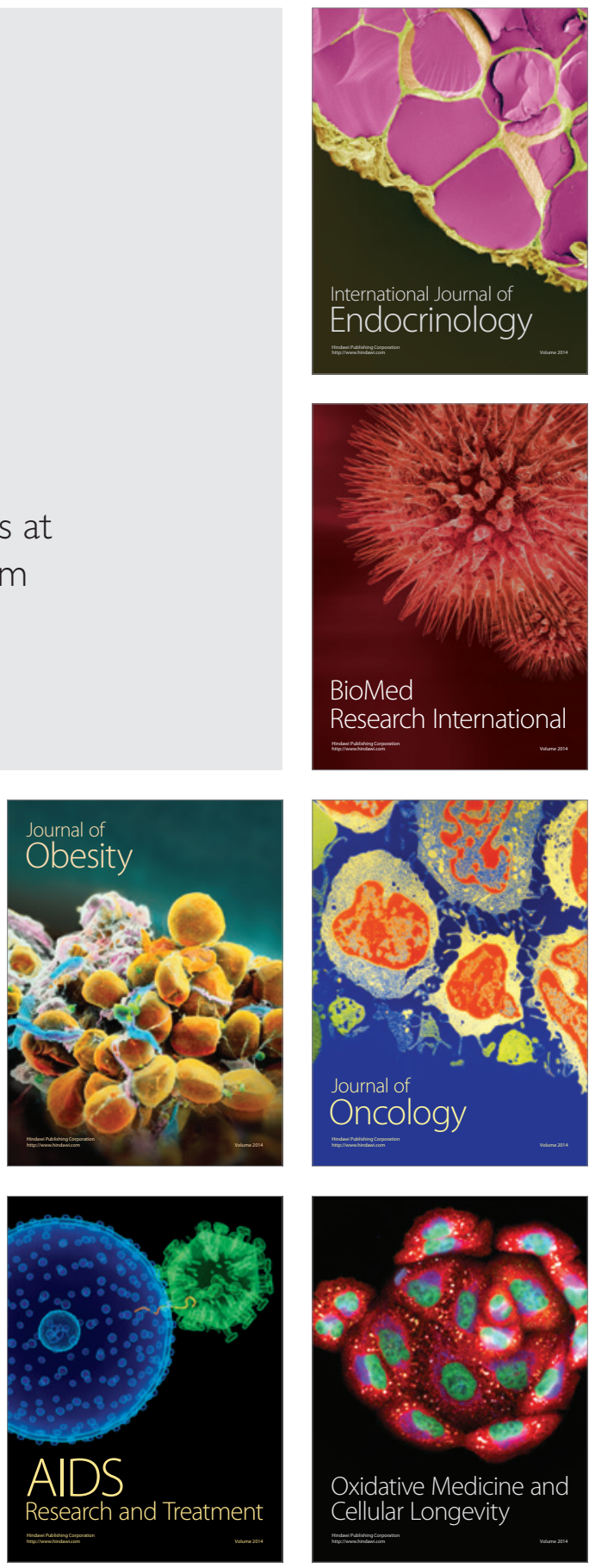